\title{
Design of a multi-energy system under different hydrogen deployment scenarios
}

\author{
Lorenzo Bartolucci ${ }^{1}$, Stefano Cordiner ${ }^{1}$, Vincenzo Mulone ${ }^{1}$, and Stefano Pasquale ${ }^{1 *}$ \\ ${ }^{1}$ University of Rome Tor Vergata, Department of Industrial Engineering, via del Politecnico 1, 00133, Rome, Italy
}

\begin{abstract}
Multi Energy Systems (MES) are effective means to increase Renewable Energy Sources (RES) penetration in the energy system and therefore to move toward a decentralized low-carbon system. Several energy vectors can be integrated together to exploit synergies in a MES framework, such as electricity, heat and hydrogen. The latter is one of the most promising energy carriers to promote widespread use of MES. Predictive management and well-defined sizing methodology are mandatory to achieve maximum performance out of MES. In this study a grid-connected MES consisting of a photovoltaic (PV) plant, a Battery Energy Storage System (BESS) and a Proton Exchange Membrane Fuel Cell (PEMFC) as a programmable Combined Cooling Heat and Power (CCHP) source, is modelled. Natural gas is considered as an alternative fuel to pure hydrogen. Mixed Integer Linear Programming and Genetic Algorithm are used respectively to solve operation and sizing problems. A single-objective optimization approach, including emission factors as optimization constraints, is carried out to find the optimal configuration of the MES. Several future scenarios are studied, considering different percentages of hydrogen in the gas mixture and comparing the techno-economic performance of the system with respect to a pure hydrogen fueling scenario. Results showed that the environmental objective within the design optimization, promote the use of hydrogen, especially in scenarios with high share of green hydrogen.
\end{abstract}

\section{Introduction}

Urban areas are high density centres of energy demand and are responsible for a large share of carbon emissions. The energy transition, which aims to keep the planet's temperature well below $2^{\circ} \mathrm{C}$ above preindustrial levels, must therefore mainly concern the urban context. However, there is a wide range of solutions to reduce the carbon footprint of urban energy systems [1].

Multi Energy Systems (MESs) are one of the most promising solutions to improve the overall sustainability of urban areas, exploiting synergies between power conversion, storage technologies and Renewable Energy Sources (RES) [2]. In urban areas, where gas and electricity networks infrastructures are developed, MES can achieve high levels of energy sectors coupling through the deployment of polygeneration concepts. Moreover, hydrogen can be integrated as an energy carrier to guarantee a higher level of integration among different sectors, improving the operation flexibility of future low carbon energy systems [3].

Due to the interaction complexity between the different components of the MES, design and energy scheduling problems can be complex tasks. Typically, the energy scheduling problem of the Energy
Management System (EMS) of a MES is solved using Mixed Integer Linear Programming (MILP), a mathematical programming technique that can rely on well-proven commercial solvers which can solve large optimization problems according to reasonable time lapses [4]. Several examples can be found in the literature [5-10]. Regarding the design task, the optimal design of a MES is strongly affected by the EMS strategy, so different approaches can be found in literature. The problem of design optimization is often addressed through the Genetic Algorithm (GA) approach [8]. A brief literature review of MES energy scheduling and design methods is presented below.

Authors in [7] employed a GA to find the storage optimal sizing of a grid-connected PV system with a rule-based operation strategy. Moreover, the authors considered an optimistic cost scenario with a $90 \%$ drop of the electrolyzer and fuel cell costs and a 50\% drop of the hydrogen storage unit. In [8] the authors proposed a bi-level optimization framework to perform an economical sizing for a stand-alone electric-only microgrid. The leader problem concerned the optimal sizing and it was solved through a GA. The follower problem regarded the energy scheduling and was solved through a MILP algorithm. Comodi et al. [9] proposed

* Corresponding author: stefano.pasquale@uniroma2.it 
an innovative methodology to design and optimize an energy system for a low-carbon community. The optimization was performed through a MILP algorithm in a purely economic scenario and in low-carbon scenarios.

It is worth noting that the economic objective is the most common in the design optimization, looking for a configuration of the energy system to minimize costs [4]. Such an approach can be too much conservative, avoiding components not yet economically competitive with the electric grid, gas grid or in general with technologies with a high Technology Readiness Level (TRL). The diffusion of hydrogen technologies, such as fuel cells and electrolyzers, is expected to grow in the next years [3], but few works have considered hydrogen-related technologies so far, within the design problem, according to market development scenarios [7]. Furthermore, fuel cells can also exploit their waste heat in cogeneration and trigeneration, coupled for example with an absorption chiller, plants to supply heating and cooling demands and increasing a MES flexibility and energy sector coupling. Loreti et al. [10] assessed the environmental and economic impact of the integration of a half-effect absorption chiller in a $50 \mathrm{~kW}$ automotive derivative PEMFC-based Combined Cooling Heat and Power (CCHP) plant fed with Natural Gas (NG). Results showed that the total cost of the system and $\mathrm{CO}_{2}$ emissions were reduced in the CCHP plant, with respect to the CHP plant, in all climatic conditions. Moreover, the interaction between pure hydrogen and the natural gas networks is expected to play a fundamental role in the decarbonization process $[3,12]$. Authors in [13] investigated the performance of a SOFC $\mu$ CHP system, fed with a blend of pure hydrogen and pure methane called Hythane. Results showed that internal reforming systems present higher thermal, electrical and overall efficiencies. Moreover, the hydrogen produced by electrolysis is also a low carbon alternative for the supply of this fuel and its production is expected to be growing rapidly [12]. In this context, hydrogen represents a fundamental energy vector, to be integrated into the MES, for a low-carbon future for urban areas.

In this work a bi-level algorithm is proposed, expressed as a leader-follower problem. The leader problem consists in the sizing optimization and it is performed through a GA that minimizes the total cost of the system. The follower problem, that consists in the dispatch optimization, is formulated with a MILP algorithm that minimizes the operational costs of the MES, based on the leader problem sizes result. The main novelty of this work is the development of a design methodology of an urban trigenerative MES that includes hydrogen market development and green hydrogen [12] deployment scenarios in the optimization process.

\section{System description and methodology}

The general MES structure considered in this work is depicted in Fig. 1. It is connected to the electric grid and gas network and equipped with heat pumps to supply heating and cooling demands. The components chosen for design optimization are the following: a PV plant; a BESS; a PEM fuel cell equipped with a fuel processor and CCHP technologies. The PEM is fuelled with natural gas taken from the grid, that is converted in hydrogen by an internal reformer. The waste heat of the FC unit is used to meet the heating demand in winter and to feed a half-effect absorption chiller in summer for the cooling demand. The PEM-CCHP can work together with the heat pumps in both seasons.

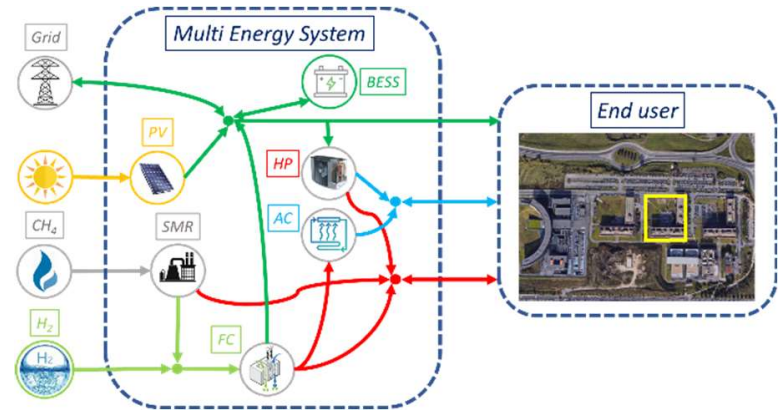

Fig. 1. General structure of the MES

\subsection{Scheduling strategy}

The results of the sizing process are strongly affected by the MES control strategy. Therefore, optimal scheduling is essential in order to optimally exploit the available technologies. The main goal of the EMS is to minimize the operating costs over a certain timespan. Since the problem variables are mixed, logical and integer, the MILP algorithm was chosen, which also represents the state-of-the-art algorithm for the scheduling of energy systems [4]. The optimization process is carried out over a one-year time horizon. The timestep used for the simulations is 8 hours. In order to express the cost function to be minimized, it is necessary to express the operating costs of each component.

\subsubsection{Battery Energy Storage System}

For the battery pack, the associated costs are mainly related to degradation and necessary replacements. The expression of costs is the same used by the authors in [8]:

$$
\begin{gathered}
C_{o p, B E S S}(t)=\frac{C_{i n v, B E S S} \cdot P_{c h}(t) \cdot \eta_{b}}{2 N}+ \\
\frac{C_{i n v, B E S S} \cdot P_{\operatorname{disch}}(t)}{2 N}
\end{gathered}
$$

Where $N$ is the maximum number of full cycles, considered equal to 4000 [8].

\subsubsection{CCHP-PEM fuel cell}

The efficiency of the trigeneration plant depends on the season. During the winter the thermal CHP efficiency is equal to 0.55 [15], while during the summer the waste heat is recovered by an absorption chiller with an average efficiency equal to 0.41 [11]. Both parameters are assumed to be constant. 


$$
Q_{C C H P}(t)=P_{P E M, e l}(t) * \eta_{t h, C C H P}(t)
$$

For the costs associated with the use of the fuel cell, the expression indicated in [8] is used and refers to maintenance, start-up and degradation.

$$
\begin{gathered}
C_{o p, P E M}(t)=\left(\frac{C_{i n v, P E M} \cdot P_{P E M, e l}(t)}{N_{P E M}}+C_{O, P E M}\right) . \\
\delta_{P E M}(t)+C_{S t a r t, P E M} \cdot \Delta \delta_{P E M}(t)
\end{gathered}
$$

\subsubsection{Fuel processing unit}

The PEM fuel cell is fed with a mixture of natural gas and hydrogen, with a hydrogen fraction in the fuel ranging between 0 and $100 \%$. For the sake of simplicity, natural gas is assumed to be composed only of pure methane. Then, the gas blend is composed of methane and hydrogen.

The $\mathrm{CO}_{2}$ emissions due to the use of the fuel cell depend on the percentage of hydrogen contained in the fuel entering the fuel processor unit. The methane fraction is processed in a Steam Methane Reformer (SMR) with an assumed emission factor of $7 \mathrm{~kg}_{\mathrm{CO} 2} / \mathrm{kg}_{\mathrm{H} 2}$ [19]. The percentage of hydrogen present in the fuel before treatment is expressed through the Hydrogen Fuel Ratio (HFR) and the emission factor of the hydrogen fraction contained in the fuel is assumed to be $2.2 \mathrm{~kg}_{\mathrm{CO} 2} / \mathrm{kg}_{\mathrm{H} 2}$, equal to the $50 \%$ of the limit imposed by the certification of hydrogen CertifHy $[12,22]$.

\subsubsection{Heat Pump}

The characteristic performance parameter of a Heat Pump is the Coefficient Of Performance (COP). The COP mainly depends on the operative conditions of the $\mathrm{HP}$, in terms of external air temperature and load factor. To avoid non-linearities within the model, the COP is considered dependent only on the external air temperature. This approximation allows to use the MILP algorithm not compromising the validity of the results. The electric power consumption of the heat pump is defined by:

$$
P_{H P}(t)=\frac{Q_{H P}(t)}{\operatorname{COP}\left(t, T_{a m b}\right)}
$$

The maximum electrical power of the heat pump is 150 $\mathrm{kW}$ while the associated operating cost is $0.2 € / \mathrm{kWh}$ [16]:

$$
\begin{gathered}
C_{o p, H P}(t)=C_{O, H P}(t) \cdot \delta_{H P}(t)+C_{S t a r t, H P} \\
\Delta \delta_{H P}(t)
\end{gathered}
$$

\subsubsection{Inequality constraints}

For the i-th component, an operating power range is considered, which takes into account the characteristics of each technology.

$$
P_{i}^{\min } \leq P_{i}(t) \leq P_{i}^{\max }
$$

Two constraints are considered to avoid the simultaneity of the charging and discharging phases, for BESS, and sale and purchase for the electricity grid:

$$
\begin{aligned}
& \delta_{C h}(t)+\delta_{\text {Disch }}(t) \leq 1 \\
& \delta_{\text {Pur }}(t)+\delta_{\text {Sell }}(t) \leq 1
\end{aligned}
$$

The SOC of the BESS is kept between the minimum and maximum values allowed to avoid aging effects:

$$
S O C_{\min }(t) \leq S O C(t) \leq S O C_{\max }(t)
$$

Some constraints are considered to prevent the BESS from charging by purchasing energy from the grid, discharging by selling energy to the grid and for the PEM to sell energy when operating in CCHP mode, to promote the self-consumption:

$$
\begin{gathered}
\delta_{C h}(t)+\delta_{\text {Pur }}(t) \leq 1 \\
\delta_{\text {Disch }}(t)+\delta_{\text {Sell }}(t) \leq 1 \\
\delta_{P E M}(t)+\delta_{\text {Sell }}(t) \leq 1
\end{gathered}
$$

\subsubsection{Equality constraints}

Two equality constraints must always be guaranteed to represent the electricity balance and the thermal balance of the MES. For the latter, the heat pump and the trigeneration system operate in parallel:

$$
\begin{gathered}
P_{\text {Disch }}(t)+P_{\text {Pur }}(t)+P_{P E M}(t)-P_{C h}(t)- \\
P_{\text {Sell }}(t)-P_{H P}(t)=P_{\text {Load }}(t)-P_{P V}(t) \\
P_{H P}(t) * C O P\left(t, T_{\text {amb }}\right)+P_{P E M}(t) * \\
\eta_{C C H P}(t)=Q_{\text {Load }}(t)
\end{gathered}
$$

\subsubsection{Emissions}

As for the fuel processing unit emission factors, the emissive parameters of each component are taken from literature. The emission factor of the electric grid, based on the Italian energy mix, is set equal to $417 \mathrm{gr}_{\mathrm{CO} 2} / \mathrm{kWh}$ [17]. Even if the operation of PV and BESS can be assumed carbon-free, emissive factors that consider their production and disposal at the end of their useful life are considered according to [20] and [21]. The emissions associated with the PEM methane-fed and hydrogen-fed are $289 \mathrm{gr}_{\mathrm{CO} 2} / \mathrm{kWh}$ and $90 \mathrm{gr}_{\mathrm{CO} 2} / \mathrm{kWh}$ respectively.

\subsubsection{MILP cost function}

The MILP objective function is expressed as follows:

$$
C_{o p}=C_{o p, B E S}+C_{o p, \text { Grid }}+C_{o p, P E M}+C_{o p, H P}
$$

MILP algorithm finds the $\min \left\{C_{o p}\right\}$. 


\subsection{Sizing algorithm}

The MES control strategy needs as input the size of the components intended as maximum power, or maximum storable energy for the BESS, of each technology. The choice of component sizes is committed to the sizing algorithm, which optimizes them based on a cost function that considers both capital and operating costs. The algorithm chosen for sizing is the GA.

\subsubsection{Capital costs}

The investment costs are considered equal to $0.66 € / \mathrm{W}_{\mathrm{p}}$ for PV [8], $250 € / \mathrm{kWh}$ for BESS [1] and $3200 € / \mathrm{kW}$ for PEM [3]. To convert the capital cost into a discounted cost, the Capital Recovery Factor (CRF) is used, expressed as:

$$
C R F=\frac{r \cdot(1+r)^{n}}{(1+r)^{n}-1}
$$

Where $r$ is the real interest rate, assumed equal to 0.05 , and $\mathrm{n}$ is the expected duration of the MES, assumed to be 20 years. The total cost is given by:

$$
\begin{gathered}
C_{c a p}=C R F \cdot\left(N_{P V} \cdot C_{i n v, P V}+C_{B E S S}\right. \\
\left.C_{i n v, B E S S} \cdot B E S S_{R e p l}+P_{P E M} \cdot C_{i n v, P E M}\right)
\end{gathered}
$$

Where $B E S S_{\text {Repl }}$ corresponds to the battery pack replacements in the MES lifetime.

\subsubsection{Maintenance costs}

The maintenance costs are assumed as in [8] and the total cost is given by:

$$
\begin{gathered}
C_{m n t}=N_{P V} \cdot C_{m n t, P V}+C_{B E S S} \cdot C_{m n t, B E S S}+ \\
P_{P E M} \cdot C_{m n t, P E M}
\end{gathered}
$$

\subsubsection{GA cost function}

Finally, the leader problem objective function can be expressed by:

$$
F=C_{c a p}+C_{o p}+C_{m n t}
$$

The leader problem is then defined by:

$$
\min _{U}\{F(U)\}
$$

\subsection{Case study}

Electric, heating and cooling loads real data are measured in the Industrial Engineering building of the University of Rome Vergata. Electric and thermal peak load are respectively $110 \mathrm{~kW}_{\mathrm{el}}$ and $390 \mathrm{~kW}_{\text {th }}$. The upper bounds for the sizes of the components are chosen based on the site's characteristics and the electrical and thermal demand profiles.

\section{Scenarios}

The MES sizing methodology that has been previously described is applied in three reference scenarios whose main characteristics are shown in Table 1. In the ECO scenario, a simple economic optimization of the MES is performed considering the current state of hydrogen technologies and related infrastructures [12].

Table 1. Scenarios simulation parameters

\begin{tabular}{|c|c|c|c|}
\hline & ECO & CER50 & CER70_HY100 \\
\hline CER [\%] & 0 & 50 & 70 \\
\hline$C_{\mathrm{H} 2}[€ / \mathrm{kg}]$ & 10 & 10 & 1 \\
\hline HFR $[\%]$ & 0 & 0 & 100 \\
\hline CPEM $[€ / \mathrm{kW}]$ & 3200 & 3200 & 800 \\
\hline
\end{tabular}

A $50 \%$ emission reduction is imposed as an environmental constraint in the CER50 scenario. The last scenario, namely CER70_HY100, concern a future ambitious projection on hydrogen technologies. In fact, the price of hydrogen and the cost of fuel cells are expected to decrease significantly from today to 2030 and beyond $[3,12]$.

\section{Results}

In this section the main results, for the three scenarios, are presented. In Fig. 2 the optimal sizes of PV, BESS and PEM fuel cell are shown. The results of the ECO scenario consist in a $113 \mathrm{~kW}_{\mathrm{p}}$ PV power plant and no BESS or PEM are considered. This is mainly due to different effects: its long useful durability; its low emission contribution compared to the grid or other components, and, finally, its high TRL which allows to achieve very low capital costs. The total cost of the system is equal to $45.11 \mathrm{k} €$ and the $\mathrm{CO}_{2}$ emissions equal to 142 tonnes. Furthermore, as highlighted by the comparison with the other two scenarios, the PV peak power is always same due to its low cost and emission intensity. On the other hand, BESS (370 kWh) and PEM $(73 \mathrm{~kW})$ are enabled in the CER50 scenario, with a total cost of $41.91 \mathrm{k} €$ and $83 \mathrm{t} \mathrm{CO}_{2}$ emissions, since the PVonly solution is no longer able to meet the emission

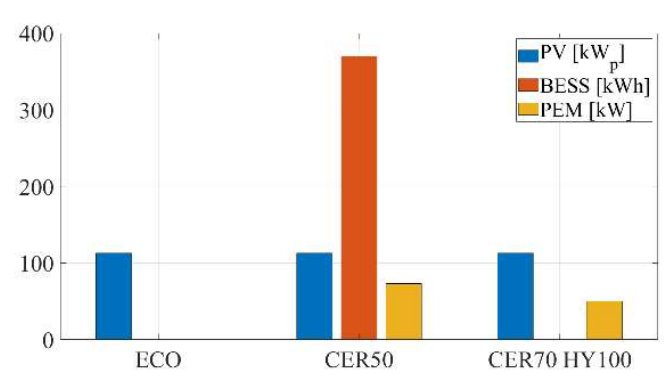

Fig. 2. Results of the sizing optimization process in the three scenarios 
constraint. In the last scenario, the PEM is considered with $50 \mathrm{~kW}$ rated power without any BESS. The cost of the system for this scenario is equal to $21.98 \mathrm{k} €$ with 21 $\mathrm{t}$ associated $\mathrm{CO}_{2}$ emissions. It is worth noting that, in comparison with ECO scenario, costs decreasing of hydrogen technologies allow to obtain significant cost reductions together with an $85 \%$ emission reduction.

The presence of BESS in the second scenario is due to the fuel cell operation. In fact, as shown in Figure 3, in the CER50 the fuel cell is activated mainly to reduce heat pumps consumption through the CCHP unit, while in the last scenario it operates for the whole year for both electric and thermal demands. Indeed, the energy purchased to total load demand ratio is considerably reduced in the latter, moving from $36 \%$ in CER50 to

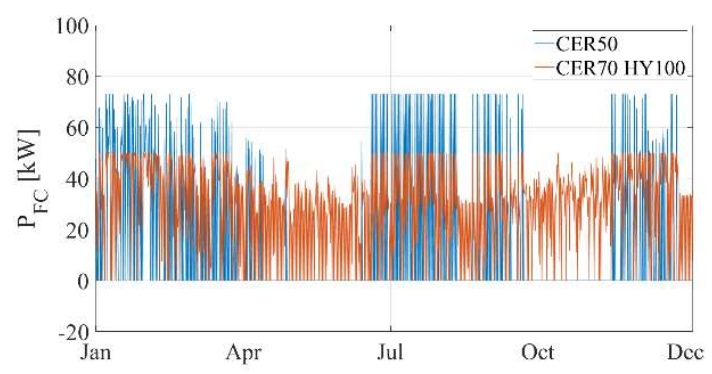

Fig. 3. Fuel cell power profiles comparison for CER50 and CER70_HY100 scenarios

about 5\% for CER70_HY100. Moreover, having considered the constraint in Eq. (12) to maximize selfconsumption, a battery pack is required in CER50 to store the surplus energy to allow the thermal-following PEM operation. On the other hand, since there is no BESS for the last scenario, the sale of energy to the grid due to the PV surplus is required. Finally, the lower emission factor associated with the hydrogen-fed PEM allows to consider a lower power size in the last scenario, keeping it in operation for most of the year up to $58 \%$ of energy share and a higher $\mathrm{CO}_{2}$ emission reduction. It is worth noting that in the winter and in the summer, whenever the thermal demand is positive, the PEM operates at rated power to fully exploit the CCHP unit, while in mid-seasons it works in part load.

In Fig. 4 the thermal profiles of the CCHP unit and the heat pumps are shown. It is worth to notice that in winter the CCHP-PEM, working at its rated power, presents thermal peak power in the same order of magnitude. On the other hand, in summer, its power output is lower than heat pumps due to the lower performance of the absorption chiller working with the waste heat. However, the CCHP unit allows to obtain

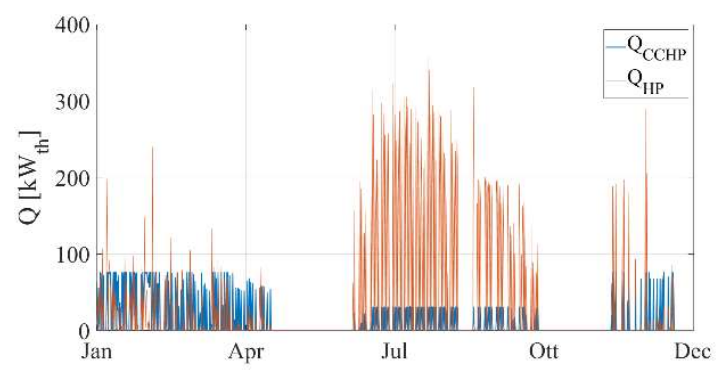

Fig. 4. Thermal power output of the CCHP system and the heat pumps in the CER70_HY100 scenario significant energy savings for heat pumps. The thermal demand percentage met by the CCHP unit is equal to about $26 \%$.

Finally, in Fig. 5 and 6, the electrical power profiles of each component for two typical weeks, respectively in the winter and the summer, are presented. In the winter case there is no surplus energy from the PV, so the energy exchanged with the grid is only purchased. The PEM almost operates to cover the baseload at rated power $(50 \mathrm{~kW})$, while the purchased energy is used for power peaks. In the summer case, with the higher PV production, the PEM still operates frequently at rated power, but several on-off switches can be also observed.

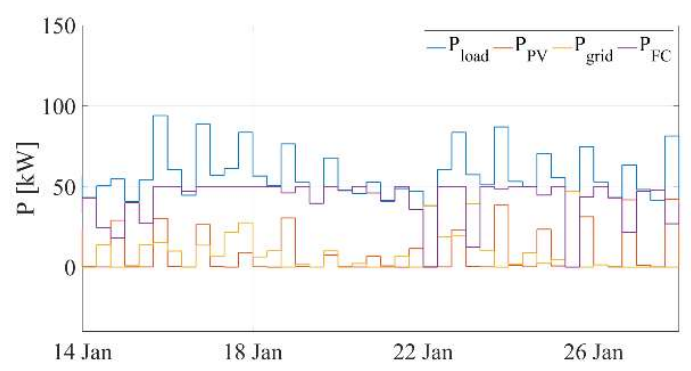

Fig. 5. Electric power profiles in two typical winter weeks in the CER70_HY100 scenario

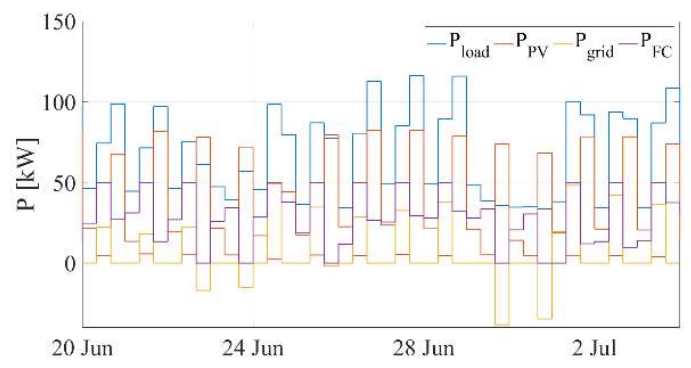

Fig. 6. Electrical power profiles in two typical summer weeks in the CER70_HY100 scenario

\section{Conclusions}

In this study a grid-connected Combined Cooling Heat and Power Multi Energy System (CCHP-MES) has been studied. Hydrogen market development and green hydrogen deployment scenarios have been included in the design methodology of the urban trigenerative MES. A Proton Exchange Membrane Fuel Cell (PEMFC) has been considered as a programmable CCHP source. To solve energy scheduling and design optimization problems a bi-level algorithm has been proposed, expressed as a leader-follower problem. The leader problem consisted in the sizing optimization and it was performed through a GA that minimizes the total cost of the system. The follower problem, that consisted in the dispatch optimization, has been formulated with a MILP algorithm that minimizes the operational costs of the MES. Different scenarios have been studied. Future projections on different percentages of hydrogen in the gas mixture, from a methane to a hydrogen network, and on its costs have been considered. Results showed that the environmental objective, expressed as an emission constraint, within the design optimization, promotes the use of hydrogen, especially in scenarios with high share 
of green hydrogen. Furthermore, in the scenario in which hydrogen is widespread, the emission constraints are observed with a significant reduction in system costs.

\section{References}

1. Sustainable, T.; Energy, U. Energy Technology Perspectives 2016 Energy Technology Perspectives 2016. 2016

2. Mancarella, P. MES (multi-energy systems): An overview of concepts and evaluation models.

Energy 2014, 65, 1-17, doi:10.1016/j.energy.2013.10.041

3. Agency, I.E. Technology Roadmap: Hydrogen and fuel cells. SpringerReference 2015, doi:10.1007/springerreference_7300

4. Gabrielli, P.; Fürer, F.; Mavromatidis, G.; Mazzotti, M. Robust and optimal design of multienergy systems with seasonal storage through uncertainty analysis. Appl. Energy 2019, 238, 1192-1210, doi:10.1016/j.apenergy.2019.01.064

5. Moser, A.; Muschick, D.; Gölles, M.; Nageler, P.; Schranzhofer, H.; Mach, T.; Ribas Tugores, C.; Leusbrock, I.; Stark, S.; Lackner, F.; et al. A MILP-based modular energy management system for urban multi-energy systems: Performance and sensitivity analysis. Appl. Energy 2020, 261, doi:10.1016/j.apenergy.2019.114342

6. Nemati, M.; Braun, M.; Tenbohlen, S. Optimization of unit commitment and economic dispatch in microgrids based on genetic algorithm and mixed integer linear programming. Appl. Energy 2018, 210, 944-963, doi:10.1016/j.apenergy.2017.07.007

7. Zhang, Y.; Campana, P.E.; Lundblad, A.; Yan, J. Comparative study of hydrogen storage and battery storage in grid connected photovoltaic system: Storage sizing and rule-based operation. Appl. Energy 2017, 201, 397-411, doi:10.1016/j.apenergy.2017.03.123

8. Li, B.; Roche, R.; Miraoui, A. Microgrid sizing with combined evolutionary algorithm and MILP unit commitment. Appl. Energy 2017, 188, 547562, doi:10.1016/j.apenergy.2016.12.038

9. Comodi, G.; Bartolini, A.; Carducci, F.; Nagaranjan, B.; Romagnoli, A. Achieving low carbon local energy communities in hot climates by exploiting networks synergies in multi energy systems. Appl. Energy 2019, 256, doi:10.1016/j.apenergy.2019.113901

10. Gabrielli, P.; Fürer, F.; Mavromatidis, G.; Mazzotti, M. Robust and optimal design of multienergy systems with seasonal storage through uncertainty analysis. Appl. Energy 2019, 238, $1192-1210$

11. Loreti, G.; Facci, A.L.; Baffo, I.; Ubertini, S. Combined heat, cooling, and power systems based on half effect absorption chillers and polymer electrolyte membrane fuel cells. Appl. Energy
2019, 235, 747-760,

doi:10.1016/j.apenergy.2018.10.109

12. Wijk, A. van; Chatzimarkakis, J. Green Hydrogen for a European Green Deal: A 2x40 GW Initiative. Hydrogen Europe. 2020, 41

13. Cinti, G.; Bidini, G.; Hemmes, K. Comparison of the solid oxide fuel cell system for micro $\mathrm{CHP}$ using natural gas with a system using a mixture of natural gas and hydrogen. Appl. Energy 2019, 238, 69-77, doi:10.1016/j.apenergy.2019.01.039

14. Yang, Y.; Zhang, H.; Yan, P.; Jermsittiparsert, K. Multi-objective optimization for efficient modeling and improvement of the high temperature PEM fuel cell based Micro-CHP system. Int. J. Hydrogen Energy 2020, 45, 69706981, doi:10.1016/j.ijhydene.2019.12.189

15. Farjah, E.; Bornapour, M.; Niknam, T.; Bahmanifirouzi, B. Placement of combined heat, power and hydrogen production fuel cell power plants in a distribution network. Energies 2012, 5, 790-814, doi:10.3390/en5030790

16. Bartolucci, L.; Cordiner, S.; Mulone, V.; Santarelli, M. Ancillary services provided by hybrid residential renewable energy systems through thermal and electrochemical storage systems. Energies 2019, 12, doi:10.3390/en12122429

17. Moro, A.; Lonza, L. Electricity carbon intensity in European Member States: Impacts intensity in European Member States: Impacts on GHG emissions of electric vehicles. Transp. Res. Part D Transp. Environ. 2018, 64, 5-14, doi:10.1016/j.trd.2017.07.012

18. Voldsund, M.; Jordal, K.; Anantharaman, R. Hydrogen production with $\mathrm{CO} 2$ capture. Int. J. Hydrogen Energy 2016, 41, 4969-4992, doi:10.1016/j.ijhydene.2016.01.009

19. Sharma, I.; Friedrich, D.; Golden, T.; Brandani, S. Exploring the opportunities for carbon capture in modular, small-scale steam methane reforming: An energetic perspective. Int. J. Hydrogen Energy 2019, 44, 14732-14743, doi:10.1016/j.ijhydene.2019.04.080

20. Frischknecht, R.; Itten, R.; Sinha, P.; WildScholten, M. de; J. Zhang, V.F.; C., H.K.; Raugei, M.; Stucki, M. Life Cycle Inventories and Life Cycle Assessment of Photovoltaic Systems; 2015; ISBN 9783906042282

21. Romare, M.; Dahllöf, L. The Life Cycle Energy Consumption and Greenhouse Gas Emissions from Lithium-Ion Batteries; 2017; ISBN 9789188319609

22. CertifHy CertifHy-SD Hydrogen Criteria, CertifHy Scheme Subsidiary Document. CertifHy Scheme Subsid. Doc. 2019 